\title{
Improvised external ventricular drain in neurosurgery: A Nigerian tertiary hospital experience
}

\author{
O. A. Ojo, M. A. Asha, O. B. Bankole, O. O. Kanu \\ Department of Surgery, Neurosurgery Unit, Lagos University Teaching Hospital, Idi-Araba, Lagos State, Nigeria
}

\begin{abstract}
Background: The most common type of hydrocephalus in developing countries is post infective hydrocephalus. Infected cerebrospinal fluid (CSF) however cannot be shunted for the reason that it will block the chamber of the ventriculo-peritoneal (VP) shunt due to its high protein content. In centers where standard external ventricular drain (EVD) sets are not available, improvised feeding tube can be used. Aim: The main focus of this study is to encourage the use of improvised feeding tube catheters for EVD when standard sets are not available to improve patients' survival. Methodology: This was a prospective study. Consecutive patients with hydrocephalus that cannot be shunted immediately for high chances of shunt failure or signs of increasing intracranial pressure were recruited into the study. Other inclusion criteria were preoperative brain tumor with possibility of blocked CSF pathway and massive intraventricular hemorrhage necessitating ventricular drainage as a salvage procedure. Standard EVD set is not readily available and too expensive for most of the parents to afford. Improvised feeding tube is used to drain/divert CSF using the standard documented procedure for EVD insertion. Outcome is measured and recorded. Results: A total of 28 patients were recruited into the study over a time frame of 2 years. There were $19(67.9 \%)$ male and $9(32.1 \%)$ females with a ratio of about 2:1. Age ranges varied from as low as 7 days to 66 years. The median age of the study sample was 6.5 months while the mean was 173.8 months. Duration of EVD varied from 2 days to 11 days with a median of 7 while the average was 6 days. Eventual outcome following the procedure of EVD showed that $19(67.9 \%)$ survived and were discharged either to go home or to have VP shunt afterwards while 8 (28.6\%) of the patients died. Conclusions: External ventricular drain can and should be done when it is necessary. Potential mortalities could be reduced by the improvised drainage using a standard feeding tube as described.
\end{abstract}

Key words: External ventricular drain, hydrocephalus, intracerebral empyema, intraventricular hemorrhage, ventriculitis

\section{Introduction}

The use of external ventricular drainage (EVD) is not new in neurosurgery, but its use in Nigeria is however not very common. The reason is due to the paucity of neurosurgical centers as well as number of surgeons in Nigeria and the fact that the standard EVD set is not readily available. Local hospital suppliers do not routinely stock or sell.

\begin{tabular}{|l|l|}
\hline \multicolumn{2}{|c|}{ Access this article online } \\
\hline Quick Response Code: & Website: \\
\hline & www.ruralneuropractice.com \\
\cline { 2 - 2 } & \\
\hline
\end{tabular}

The main function of EVD is essentially to drain the ventricle howbeit temporarily. In few cases, it may be necessary for $>1$ week. On specific occasions, it may be used as a portal for intraventricular antibiotics. The general reasoning is to leave it in situ until it is no more necessary. Few patients require long term drainage of their cerebrospinal fluid (CSF) and are thereafter shunted. ${ }^{[1]}$

External ventricular drain is a routine procedure in major pediatric and adult neurosurgical services. Likely indications include traumatic intraventricular hemorrhage (IVH), infected CSF precluding shunting and after a posterior fossa surgery as a prophylactic channel for control of intracranial pressure from possible blockage of aqueduct of sylvius. ${ }^{[1-3]}$

We document our experience with the use of EVD despite the challenges from procuring the standard

Address for correspondence:

Mr. Omotayo A. Ojo, Department of Surgery, Neurosurgery Unit, Lagos University Teaching Hospital, PMB 12007, Surulere, Idi-Araba, Lagos State, Nigeria. E-mail: tayoojo111@yahoo.com 
EVD set and adaptations we made to overcome the local limitations.

The aim of the study is to create awareness that EVD is helpful in selected cases and can be done in emergent situations even when the ideal set is not available.

\section{Methodology}

The study period was from January 2012 to December 2013 , a time frame of 2 years. The decision to externally divert the CSF is clinical and made by the attending neurosurgeon. This decision is based on clinical presentation of the patient, physical examination and radiologic as well as laboratory findings of CSF abnormalities. All patients with salvageable intraventricular bleed were recruited into the study. Patient with a Glasgow coma score (GCS) above 6, with reactive pupils (with or without anisocoria) were recruited while patients with dilated and fixed pupils following intraventricular bleed were not recruited.

All pediatric patients with hydrocephalus and documented infected CSF were included in the study. When children present with tense anterior fountanel, craniofacial disproportion, cloudy CSF on ventricular tap to reduce increased intracranial pressure, culture positive of same CSF, raised white blood cell count and differentials suggestive of active infective process, they were included in the study.

Patients (adults or children) who were to have posterior fossa surgery for brain tumor had an EVD inserted as a prophylactic procedure.

A size 6 or 8 (French guage) feeding tube [Figure 1] is used as the EVD catheter for the simple reason that it is cheap and readily available while at the same time the ideal set is scarce and expensive. Size 6 is usually used to drain infected CSF while size 8 is used in cases of IVH.

Once the decision to do EVD is made, patients are made to sign an informed consent with the details of the procedure explained by the consultant. The possible complications as well as the benefits are clearly explained to the patient or the relative in instances where the patient is unable to sign the consent. The procedure is done in the theatre under general anesthesia except a few cases where the patient is so unstable that he/she could not wait for any delay in the procedure, it is then done by the bedside under strict aseptic procedure and local anesthesia.
The usual drainage point is the right frontal horn, and the procedure is done without any image guidance but by a freehand method. ${ }^{[4]} \mathrm{A}$ burr hole is made at the point 2-3 cm lateral to the sagittal suture and 1-2 cm anterior to the coronal suture (Kocher's point) on the right. The procedure is done using the established landmarks to the frontal horn especially cannulating the ventricle with a trajectory that is perpendicular to the skull. ${ }^{[5]}$ The feeding tube is advanced up to $5-6 \mathrm{~cm}$ from the dura and the tube is tunneled to about $5-7 \mathrm{~cm}$ away from the burr hole point to reduce the chances of infection. CSF is diverted through an infusion set into a sterile collection bag and allowed to drain without a vacuum attached. A sterile urine collection bag is often improvised for the collection bag [Figure 2]. This is a closed drainage set to ensure sterility of the procedure. In hemorrhagic stroke with IVH, the EVD is placed on the contralateral side to the IVH or the less affected side.

Routine collection of CSF for CSF protein, microscopy, culture and sensitivity were done at regular intervals from the EVD to ascertain clearing of infection and possible readiness for shunting procedure. ${ }^{[6]}$ Strict asepsis is maintained during CSF sampling.

Prior to removal of EVD, the drain is challenged for $24 \mathrm{~h}$ to be sure the patient is not drain dependent. This is done by raising the EVD tube column to at least $20 \mathrm{~cm}$ above the external auditory meatus with the head in neutral position, the drain is then clipped for another $24 \mathrm{~h}$ before final removal if the patient remains stable.

All patients had prophylactic antibiotic cover (intravenous [IV] ceftriaxone $50 \mathrm{mg} / \mathrm{kg}$ body weight) before the insertion of EVD. Patient with infected CSF were on specific IV antibiotic for 4 weeks and continued on oral antibiotics for 2 more weeks.

All patients included in the study had the bio-data entered into the database. Date of procedure, duration of the EVD, indication for EVD, culture result of CSF, outcome following the EVD and a comment of its advantage in helping the patient.

The data were processed and analyzed.

\section{Results}

A total of 28 patients were recruited into the study within the time frame of 2 years. They were consecutive patients that met the inclusion criteria. There were 19 (67.9\%) male and 9 (32.1\%) females with a ratio of about 2:1. Age ranges varied from as low as 7 days to 66 years. The 
median age of the study sample was 6.5 months while the mean was 173.8 months.

Indications for EVD included brain tumor especially for ventricular drainage in posterior fossa surgery, hemorrhagic CSF with hydrocephalus, infected CSF and ventriculitis with associated hydrocephalus and stroke with associated IVH + intracerebral hemorrhage. Hemorrhagic CSF and infected CSF accounted for $7(23.3 \%)$ and $9(30 \%)$ indications for EVD respectively. Of the infected CSF patients, 2 patients $(7.1 \%)$ had frank pus drained at insertion of EVD. Other indications for EVD in this study especially in the older patients are stroke with associated intraventricular and intraparenchyma hemorrhage accounting for $6(21.4 \%)$ patients.

Duration of EVD varied with the indications for the procedure. The range was from 2 days to 11 days with a median of 7 while the average was 6 days.

Eventual outcome following the procedure of EVD showed that 19 (67.9\%) survived and were discharged either to go home or to have ventriculo-peritoneal (VP) shunt afterwards while $8(28.6 \%)$ of the patients died. One patient was discharged against medical advice by the family and was lost to follow-up.

Outcome based on shunt drain dependency showed that $8(28.6 \%)$ patients did not require further CSF diversions when the drain was removed while $11(39.3 \%)$ of the patients had to be shunted when the CSF was clear and protein level within normal range for shunting procedure. 1 (3.6\%) patient was referred out from our center and was not followed-up.

Though the procedure was done using a freehand technique, there was no further neurologic deficit attributable to the procedure. In $5(17.9 \%)$ of patients,

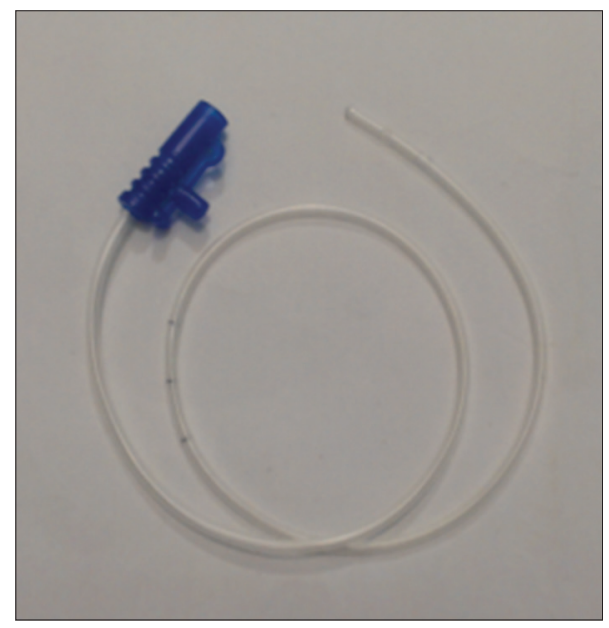

Figure I: Size six feeding tube there were drain site infection and these settled within few days while on antibiotics. All patients who had superficial drain site infection already had ventriculitis with or without frank pus drained.

The main indications for EVD were to temporarily drain CSF in patients with hydrocephalus that could not immediately be shunted because of infected CSF and, peri-operatively for patients with posterior fossa tumor or hemorrhagic CSF. Twenty-one (75.0\%) patients had EVD for the purpose of drainage alone while in $4(14.3 \%)$ patients; the use included antibiotic administration to the intraventricular space in addition to temporary CSF drainage. Only in $3(10.7 \%)$ patients was the use for drainage of blood.

\section{Discussions}

The important finding in this study is that EVD can be done with improvised tube when the standard set is not available, especially in resource poor centers. EVD is a life-saving procedure used to divert CSF in hydrocephalus when there are reasons why VP shunt could not be done immediately and also as a temporary and prophylactic procedure in posterior fossa surgery as in some of the cases in this study. ${ }^{[3]}$ In emergent instances like infected or hemorrhagic hydrocephalus, the procedure is safe to be done by the bed side and using a freehand method..$^{[4,5,7]}$ Where image guidance is available, it may improve the accuracy of placement in the frontal horn of lateral ventricle. ${ }^{[8]}$

In infected and hemorrhagic CSF, EVD has the additional advantage of being a portal for intraventricular antibiotic administration while draining infected CSF [Figure 3]. 4 of the patients in the study benefited from this advantage. Its use is also encouraged to reduce intracranial pressure and divert CSF in IVH [Figure 4a].

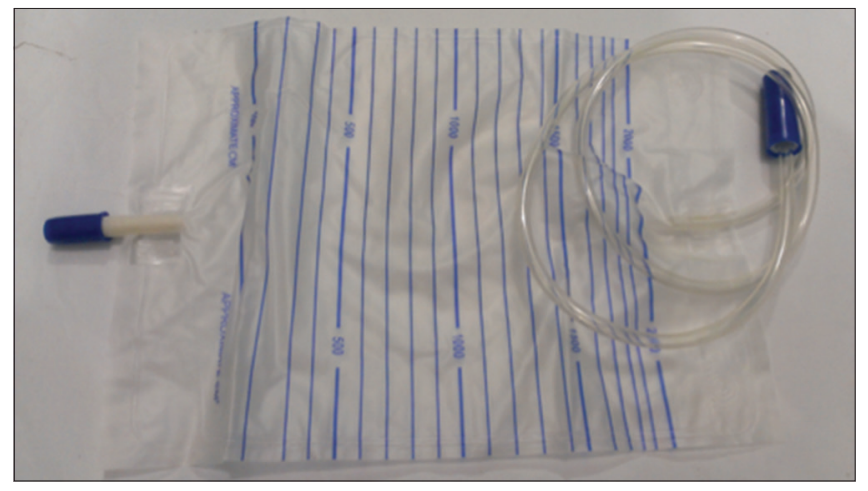

Figure 2: Standard sterile collection bag 


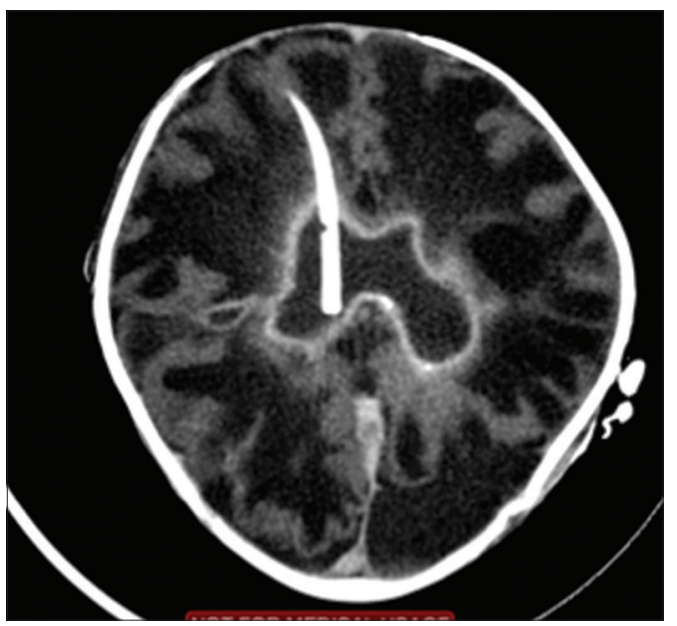

Figure 3: External ventricular drain in the ventricle draining infected cerebrospinal fluid

The cost of a standard EVD set made by Codman ${ }^{\circledR}$ is about $\$ 45$ on ebay. ${ }^{[9]} \mathrm{A}$ feeding tube and standard draining bag as shown in Figures 1 and 2 cost less than $\$ 5$ locally. In countries with health insurance, such a set is readily available to patients in emergency settings at no immediate cost to the patient. Unfortunately, medical services have to be paid for out of pocket in our region. ${ }^{[10]}$ Resources are limited as well, and materials for such services are not readily available in developing countries. We therefore set out to use a simple and efficient alternative. The aim is not to downplay the importance of the standard set, but to offer help in the midst of the apparent lack and want.

All the indications for EVD in this study are life threatening, and patients could have a high rate of fatality if nothing is done or while waiting for ideal EVD set. With a survival of $19(67.9 \%)$ patients out of 28 , it is worth giving patients that require urgent CSF diversion a chance for survival. There is no doubt of its usefulness in neurosurgery practice with regards to patients with potential fatal fate from increased intracranial pressure either from hydrocephalus, IVH/SAH or brain tumor. ${ }^{[1,11]}$

As expected, the patient population is sharply divided into the adult and the children. The indication in children was essentially from hydrocephalus of different causes and posterior fossa tumor while in adults it was essentially from cerebrovascular causes and few brain tumors. ${ }^{[1,12]}$ Studies elsewhere also documented such variations in indication for EVD and age distributions. The main reason in IVH is not to drain the blood in the ventricle but to drain CSF and prevent hydrocephalus. Invariably, there will be some drainage of blood in the ventricle. The EVD is therefore inserted in the contralateral side to the IVH [Figure 4a-c].

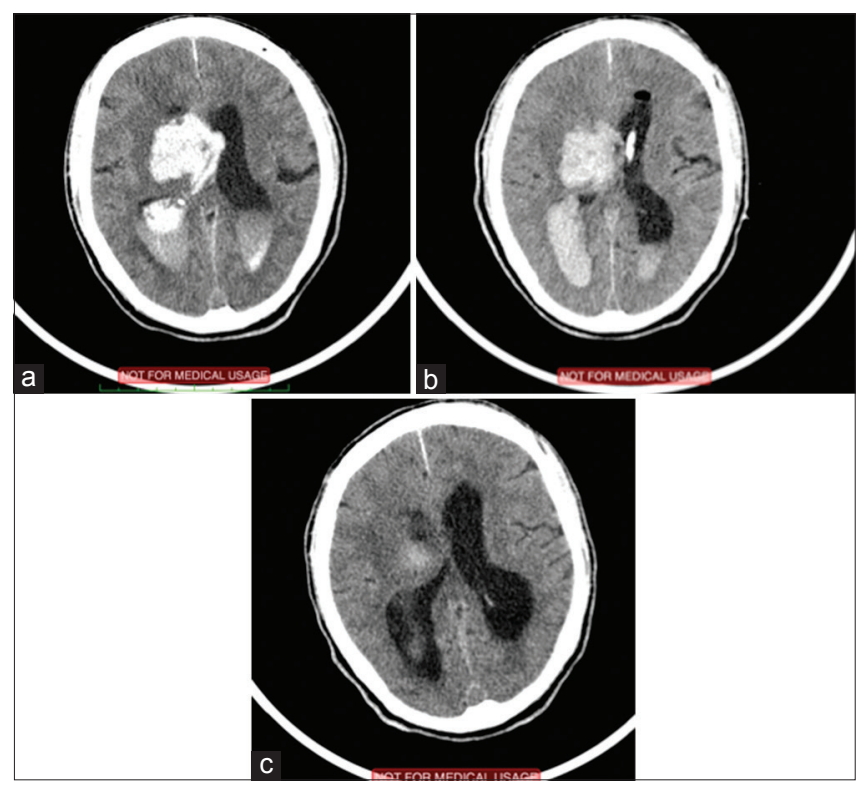

Figure 4: (a) Preoperative image showing intracerebral hemorrhage (ICH) and intraventricular hemorrhage (IVH) (b) Image immediately after external ventricular drain (EVD) insertion. Tube is in the left ventricle (c) computed tomography of the same patient after 2 weeks post ICH/IVH and EVD

Blood in the ventricle may of course form clots with the tendency to block the aqueduct of Sylvius. It is possible that it is the accumulation of CSF (hydrocephalus) that adds to the already increased intracranial pressure that contributes to the demise of stroke patients. Diverting the CSF and blood outside the brain howbeit temporarily may increase the chances of survival for such patients.

Duration of EVD is also variable. The ideal antibiotic impregnated EVD set could be in situ for $>10$ days, whereas the plain EVD set is advised to be removed within 5 days. There are studies that showed reduced rate of infected EVD with antibiotic impregnated EVD sets. ${ }^{[13]}$ There is no specific time to discontinue EVD and such decisions are taken on individual patient basis. It is always important to challenge the EVD either through the use of a transducer, which is not available in our practice or by manometry as described earlier. When the EVD is challenged, we watch out for deterioration in the level of consciousness in children and increasing headache with drop in GCS in adults. Only when patients do well over $24 \mathrm{~h}$ were we encouraged to remove the EVD.

In our study, the average duration was 6 days with the improvised EVD set. Patient who had EVD as a prophylaxis after a tumor surgery did not require it for long. EVD for posterior fossa tumor surgery is to prevent acute hydrocephalus from obstruction of the aqueduct of Sylvius and also from possibility of edema of the 
cerebellum causing obstruction to CSF outflow. This could rapidly be life-threatening hence the use of EVD.

The most common complication associated with EVD is infection of the set. It is responsible for the morbidity and mortalities associated with the procedure. Though we observed EVD site infection in 5 of cases, there is no certainty as regards the culpability of the procedure. In such cases, the CSF to be drained was already infected, and there is a possibility that the same organism was responsible. Such superficial infections resolved with antibiotic. A protocol clearly written for insertion and care of patients with EVD would help in reducing chances of EVD infections. ${ }^{[14-16]}$

There have been various discussions relating to the effect of length of drainage and infection rates. Though most are in support of changing or removing the EVD set within 5 days, there is no conclusive evidence that length of drainage increases the chances of EVD infection. ${ }^{[12,17]}$ The EVD set was left for as long as needed, and there are evidence to suggest this is a good practice in a randomized study done by Wong et al. ${ }^{[17]}$ and others. ${ }^{[12,18]}$

Tunneling the EVD tube away from the burr hole site is a good measure to prevent EVD site infection and ventriculitis. All the patients in the study had EVD tunneled at least $5-7 \mathrm{~cm}$ away from incision for burr hole site. There are studies that agree with this practice. ${ }^{[19]}$

Rarer complications of EVD insertion include upward gaze palsy, ${ }^{[20]}$ vascular incidence, ${ }^{[21]}$ tension pneumocephalus ${ }^{[3]}$ among others. These had been documented in various case reports. They are not common, and we did not experience any of such complications despite using an improvised set.

\section{Conclusion}

We have shown that using an appropriately sized feeding tube as an improvised EVD is safe, associated with minimal complications and can help save lives in critically ill patients who otherwise would have a dismal outcome in our environment.

\section{References}

1. Walker CT, Stone JJ, Jacobson M, Phillips V, Silberstein HJ. Indications for pediatric external ventricular drain placement and risk factors for conversion to a ventriculoperitoneal shunt. Pediatr Neurosurg 2012;48:342-7.

2. Patil V, Lacson R, Vosburgh KG, Wong JM, Prevedello L, Andriole K, et al. Factors associated with external ventricular drain placement accuracy: Data from an electronic health record repository. Acta Neurochir (Wien) 2013;155:1773-9.

3. Prabhakar H, Ali Z, Rath GP, Bithal PK. Tension pneumocephalus following external ventricular drain insertion. J Anesth 2008;22:326-7.

4. Hsieh CT, Chen GJ, Ma HI, Chang CF, Cheng CM, Su YH, et al. The misplacement of external ventricular drain by freehand method in emergent neurosurgery. Acta Neurol Belg 2011;111:22-8.

5. Muirhead WR, Basu S. Trajectories for frontal external ventricular drain placement: Virtual cannulation of adults with acute hydrocephalus. Br J Neurosurg 2012;26:710-6.

6. Wong FW. Cerebrospinal fluid collection: A comparison of different collection sites on the external ventricular drain. Dynamics 2011;22:19-24.

7. Phillips SB, Delly F, Nelson C, Krishnamurthy S. Bedside external ventricular drain placement: Can multiple passes be predicted on the computed tomography scan before the procedure? World Neurosurg 2014;82:739-44.

8. Chai FY, Farizal F, Jegan T. Coma due to malplaced external ventricular drain. Turk Neurosurg 2013;23:561-3.

9. Available from: http://www.ebay.com/itm/Codman-EVD-Bactiseal-CathSet-1-9 mmx35 cm-w-Stylet-Trocar-82-1749-/290962903774?pt=LH_ DefaultDomain_0 and hash=item43bebd92de. [Last accessed on 2014 Dec 17].

10. Kanu OO, Nnoli C, Olowoyeye O, Ojo O, Esezobor C, Adeyomoye A, et al. Infantile subdural empyema: The role of brain sonography and percutaneous subdural tapping in a resource-challenged region. J Neurosci Rural Pract 2014;5:355-9.

11. Bhargava D, Alalade A, Ellamushi H, Yeh J, Hunter R. Mitigating effects of external ventricular drain usage in the management of severe head injury. Acta Neurochir (Wien) 2013;155:2129-32.

12. Roitberg BZ, Khan N, Alp MS, Hersonskey T, Charbel FT, Ausman JI. Bedside external ventricular drain placement for the treatment of acute hydrocephalus. Br J Neurosurg 2001;15:324-7.

13. Muttaiyah S, Ritchie S, John S, Mee E, Roberts S. Efficacy of antibiotic-impregnated external ventricular drain catheters. J Clin Neurosci 2010;17:296-8.

14. Rivero-Garvía M, Márquez-Rivas J, Jiménez-Mejías ME, Neth O, Rueda-Torres AB. Reduction in external ventricular drain infection rate. Impact of a minimal handling protocol and antibiotic-impregnated catheters. Acta Neurochir (Wien) 2011;153:647-51.

15. Dasic D, Hanna SJ, Bojanic S, Kerr RS. External ventricular drain infection: The effect of a strict protocol on infection rates and a review of the literature. Br J Neurosurg 2006;20:296-300.

16. Lwin S, Low SW, Choy DK, Yeo TT, Chou N. External ventricular drain infections: Successful implementation of strategies to reduce infection rate. Singapore Med J 2012;53:255-9.

17. Wong GK, Poon WS, Wai S, Yu LM, Lyon D, Lam JM. Failure of regular external ventricular drain exchange to reduce cerebrospinal fluid infection: Result of a randomised controlled trial. J Neurol Neurosurg Psychiatry 2002;73:759-61.

18. Korinek AM, Reina M, Boch AL, Rivera AO, De Bels D, Puybasset L. Prevention of external ventricular drain - Related ventriculitis. Acta Neurochir (Wien) 2005;147:39-45.

19. Rafiq MF, Ahmed N, Ali S. Effect of tunnel length on infection rate in patients with external ventricular drain. J Ayub Med Coll Abbottabad 2011;23:106-7.

20. Rosenbaum BP, Wheeler AM, Krishnaney AA. External ventricular drain placement causing upgaze palsy: Case report. Clin Neurol Neurosurg 2013;115:1514-6.

21. Kosty J, Pukenas B, Smith M, Storm PB, Zager E, Stiefel M, et al. Iatrogenic vascular complications associated with external ventricular drain placement: A report of 8 cases and review of the literature. Neurosurgery 2013;72:ons208-13.

How to cite this article: Ojo OA, Asha MA, Bankole OB, Kanu OO. Improvised external ventricular drain in neurosurgery: A Nigerian tertiary hospital experience. J Neurosci Rural Pract 2015;6:304-8.

Source of Support: Nil. Conflict of Interest: None declared. 